\title{
Approximate Object Location and Spam Filtering on Peer-to-Peer Systems
}

\author{
Feng Zhou, Li Zhuang, Ben Y. Zhao, Ling Huang, \\ Anthony D. Joseph, and John Kubiatowicz \\ Computer Science Division, U. C. Berkeley \\ $\{z f, z l$, ravenben, hling, adj,kubitron\}@cs.berkeley.edu
}

\begin{abstract}
Recent work in P2P overlay networks allow for decentralized object location and routing (DOLR) across networks based on unique IDs. In this paper, we propose an extension to DOLR systems to publish objects using generic feature vectors instead of content-hashed GUIDs, which enables the systems to locate similar objects. We discuss the design of a distributed text similarity engine, named Approximate Text Addressing (ATA), built on top of this extension that locates objects by their text descriptions. We then outline the design and implementation of a motivating application on ATA, a decentralized spam-"Itering service. We evaluate this system with 30,000 real spam email messages and 10,000 non-spam messages, and "nd a spam identi"cation ratio of over $97 \%$ with zero false positives.
\end{abstract}

Keywords: Peer-to-peer, DOLR, Tapestry, spam "ltering, approximate text matching

\section{Introduction}

Recent work on structured P2P overlay networks ([5.18], [15], [11], [10]) utilize scalable routing tables to map unique identi“ers to network locations, providing interfaces such as Decentralized Object Location and Routing (DOLR) and Distributed Hashtables (DHT). They allow network applications such as distributed "le systems and distributed web caches to ef"ciently locate and manage object replicas across a wide-area network.

While these systems excel at locating objects and object replicas, they rely on known Globally Unique IDenti“"ers (GUID) for each object, commonly generated by applying a secure hash function to the object content. This provides a highly speci“c naming scheme, however, and does not lend itself to object location and management based on semantic features.

To address this problem, we propose an approximate location extension to DOLR systems to publish and locate objects using generic feature vectors composed of a number of values generated from its description or content. Any object can be addressed by a feature vector matching a minimal threshold number of entries with its original feature vector. Based on this extension, we propose an Approximate Text Addressing (ATA) facility, which instantiates the approximate location extension by using block text "ngerprints as features to "nd matches between highly similar text documents. To validate the ATA design as well as the approximate object location extension, we design a decentralized spam-"Itering application that leverages ATA to accurately identify junk 
email messages despite formatting differences and evasion efforts by spammers. We evaluate the accuracy of our "ngerprint vector scheme via simulation and analysis on real email data, and explore the trade-offs between resource consumption and search accuracy.

The rest of this paper is as follows: Section 2 brie"y describes existing work in P2P overlays. Section 3 presents our approximation extension to DOLR systems and a prototype implementation. Section 4 describes the design of ATA and Section 5 discusses the design of the decentralized spam "lter. Section 6 presents simulation and experimental results, followed by a discussion of related work in Section 7 and status and future work in Section 8. Finally, we provide a mathematical analysis of the robustness of text-based "ngerprinting in Appendix A.

\section{Background: Structured P2P Overlays}

In this section, we "rst present background material on structured P2P overlays. Different protocols differ in semantics details and performance objectives. While we present our work in the context of Tapestry for performance reasons, our design is general, and our results can be generalized to most structured $\mathrm{P} 2 \mathrm{P}$ protocols.

\subsection{Routing}

Tapestry is an overlay location and routing layer "rst presented in [18], with a rigorous treatment of dynamic algorithms presented in [5]. Like other structured P2P protocols, object and node IDs are pseudo-randomly chosen from the namespace of "x ed-length bit sequences with a common base (e.g. Hex). Tapestry uses local routing tables at each node to route messages incrementally to the destination ID digit by digit (e.g., $4 \star \star \star \Longrightarrow$ $45 * \star \Longrightarrow 459 * \Longrightarrow 4598$ where * ${ }^{*}$ s represent wildcards). A node $N$ has a neighbor map with multiple levels, where each level represents a matching pre" $x$ up to a digit position in the ID. Each level of the neighbor map contains a number of entries equal to the base of the ID, where the $i^{\text {th }}$ entry in the $j^{\text {th }}$ level is the location of the node closest in network latency that begins with prefix $x_{j-1}(N)+i$.

To forward on a message from its $n^{t h}$ hop router, Tapestry examines its $n+1^{t h}$ level routing table and forwards the message to the link corresponding to the $n+1^{\text {th }}$ digit in the destination ID. This routing substrate provides ef" cient location-independent routing within a logarithmic number of hops and using compact routing tables. Figure 1 shows a Tapestry routing mesh.

\subsection{Data Location}

In Tapestry, a server $S$ makes a local object $O$ available to others by routing a $\bullet$ publish $Z \check{~}$ message to the object•s $\bullet$ root node, $\breve{Z}$ the live node $O \bullet$ s identi“"er maps to. At each hop along the path, a location mapping from $O$ to $S$ is stored. Mappings for multiple replicas are stored sorted according to distance from the local node. See Figure 2 for an example of object publication. Here two replicas of the same object are published. A client routes a query message towards the root node. The message queries each hop router along the 


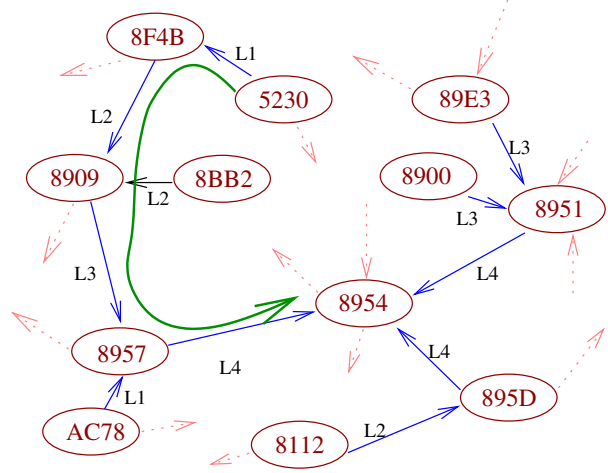

Fig. 1. Tapestry routing example. Path taken by a message from node 5230 for node 8954 in Tapestry using hexadecimal digits of length 4 (65536 nodes in namespace).

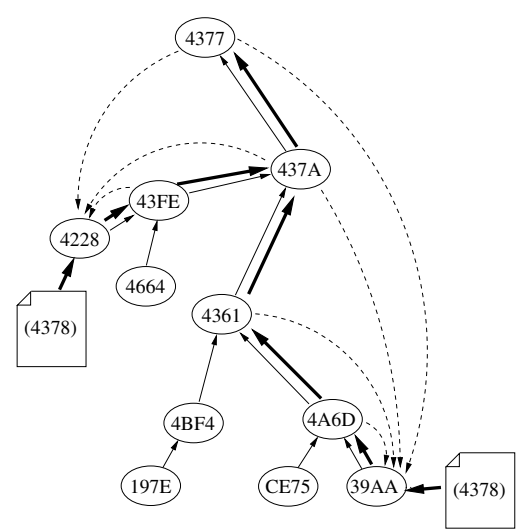

Fig. 2. Publication in Tapestry. To publish object 4378 , server 39AA sends publication request towards root, leaving a pointer at each hop. Server 4228 publishes its replica similarly. Since no 4378 node exists, object 4378 is rooted at node 4377 .

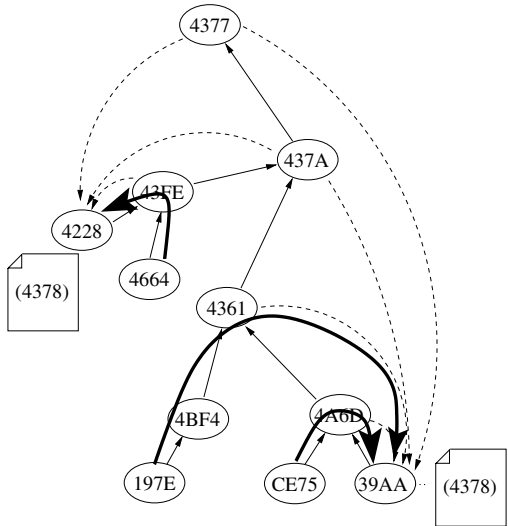

Fig. 3. Object Location in Tapestry: Three different location requests. For instance, to locate GUID 4378 , query source $197 \mathrm{E}$ routes towards the root, checking for a pointer at each step. At node 4361 , it encounters a pointer to server 39AA.

way, and routes towards $S$ when it nds the $O$ to $S$ location mapping. Note that for nearby objects, query messages quickly intersect the path taken by publish messages, resulting in quick search results that exploit locality [18]. See Figure 3 for an example of object location. Notice how locality is exploited by directing location requests to nearby replicas.

\section{Approximate DOLR}

DOLR systems like Tapestry provide deterministic, scalable, and ef cient location and routing services, making them attractive platforms for deploying wide-area network 
applications. Files, in particular, can be located ef"ciently if their canonical name is known. Previous approaches, however, generate Globally Unique IDenti"ers (GUID) by a secure hash (e.g. SHA-1) of the content. This approach signi“cantly limits the usability of the system in scenarios where users do not known exact names of objects, but rather perform searches based on general characteristics of the system. In particular, these scenarios might include searches for data that closely approximates, or is similar to known data with certain properties. Examples might include searching for audio or video that matches existing works in content features, or searching or lightly modi“"ed replicas of existing data.

\subsection{Approximate DOLR Design}

Here we propose an extension to DOLR, Approximate DOLR, as a generic framework to address some of the needs of these applications. In an ADOLR system, we apply application-speci"c analysis to given objects to generate feature vectors that describe its distinctive features, and provide a translation mechanism between these applicationdriven features and a traditional GUID obtained from a secure content hash of the object contents.

This query ability on features applies to a variety of contexts. In the world of multimedia search and retrieval, we can extract application-speci“c characteristics, and hash those values to generate feature vectors. Any combination of "eld to value mappings can be mapped to a feature vector, given a canonical ordering of those "elds. For example, this can be applied to searching for printer drivers given printer features such as location, manufacturer, and speed. If features are canonically ordered as [location, manufacturer, speed], then an example feature vector might be [hash ( 443 Soda), hash(HP), hash(12ppm) ].

Each member of the vector, a feature, is an application-speci"c feature encoded as a hashed identi"er. For each feature $f$, an object (feature object) is stored within the network. The feature object is a simple object that stores the list of GUIDs of all objects whose feature vectors include $f$. Clients searching for objects with a given feature set "nds a set of feature objects in the network, each associated with a single feature, and selects the GUIDs which appear in at least $T$ feature objects, where $\mathrm{T}$ is a tunable threshold parameter used to avoid false positives while maintaining the desired generality of matches.

The •publicationŽ of an object $O$ in an ADOLR system proceeds as follows. First, its content-hash derived GUID is "rst published using the underlying P2P DOLR layer. This assures that any client can route messages to the object given its GUID. Next, we generate a feature vector for $O$. For each feature in the vector, we try to locate its associated feature object. If such an object is already available in the system, we append the current GUID to that object. Otherwise, we create a new feature object identi"ed by the feature, and announce its availability into the overlay.

To locate an object in an ADOLR system, we "rst retrieve the feature object associated with each entry of the feature vector. We count the number of distinct feature objects each unique GUID appears in, and select the GUID(s) that appear in a number greater than some preset threshold. The GUID(s) are then used to route messages to the desired object. 
The ADOLR API is as follows:

- PublishApproxObject (FV, GUID). This publishes the mapping between the feature vector and the GUID in the system. A feature vector is a set of feature values of the object, whose de"nition is application speci"c. Later, one can use the feature vector instead of the GUID to search for the object. Notice that PublishApproxObject only publishes the mapping from FV to GUID. It does not publish the object itself, which should be done already using publish primitive of Tapestry when PublishApproxObject is called.

- UnpublishApproxObject (FV, GUID). This removes the mapping from the FV to the GUID if this mapping exists in the network, which is the reverse of PublishApproxObject.

- RouteToApproxObject (FV, THRES, MSG). This primitive routes a message to the location of all objects which overlap with our queried feature vector FV on more than THRES entries. The basic operations involve for each feature, retrieving a list of GUIDs that share that feature, doing a frequency count to "lter out GUIDs that match at least THRES of those features, and "nally routing the payload message MSG to them. For each object in the system with feature vector $F V^{*}$, the selection criterion is:

$$
\left|F V^{*} \bigcap F V\right| \geq T H R E S \text { AND } 0<T H R E S \leq|F V|
$$

The location operation is deterministic, which means all existing object IDs matching the criterion will be located and be sent the payload message. However, it is important to notice that this does not mean every matching object in the system will receive the message, because each object ID may correspond to multiple replicas, depending on the underlying DOLR system. The message will be sent to one replica of each matching object ID, hopefully a nearby replica if the DOLR utilizes locality.

With this interface, we reduce the problem of locating approximate objects on $\mathrm{P} 2 \mathrm{P}$ systems to "nding a mapping from objects and search criteria to feature vectors. The mapping should maintain similarity relationships, such that similar objects are mapped to feature vectors sharing some common entries. We show one example of such a mapping for text documents in Section 4.

\subsection{A Basic ADOLR Prototype on Tapestry}

Here we describe an Approximate DOLR prototype that we have implemented on top of the Tapestry API. The prototype serves as a proof of concept, and is optimized for simplicity. The prototype also allows us to gain experience into possible optimizations for performance, robustness and functionality.

The prototype leverages the DOLR interface for publishing and locating objects, given an associated identi“er. When PublishApproxObject is called on an object $O$, it begins by publishing $O \bullet$ s content-hashed object GUID using Tapestry. Then the client node uses Tapestry to send messages to all feature objects involved. Tapestry routes these messages to the nodes where these feature objects are stored. These nodes then add the new object GUID to the list of GUIDs inside the feature object. If any feature object 


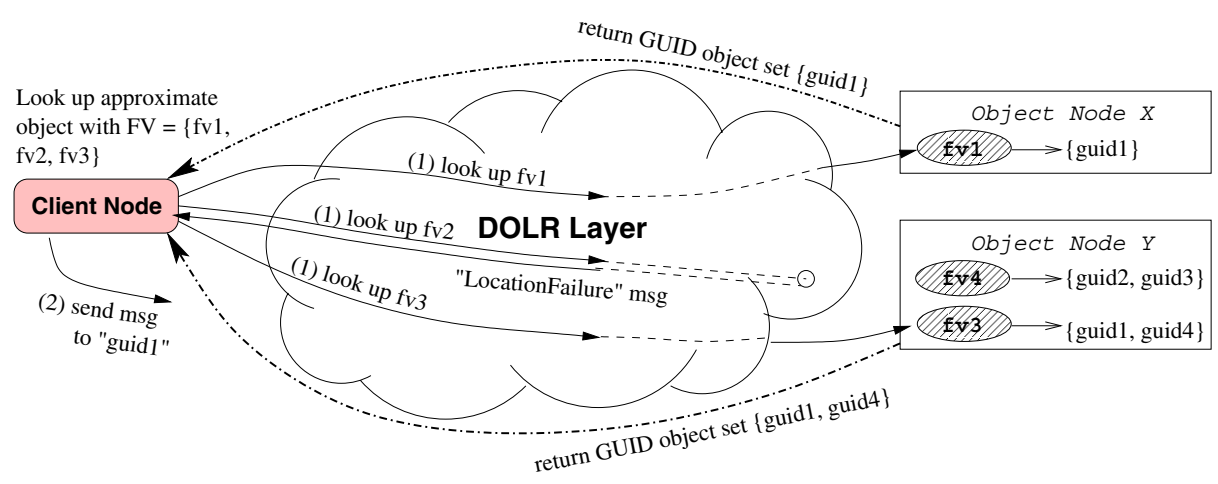

Fig. 4. Location of an approximate object. Client node wants to send a message to all objects with at least 2 feature in $\{\mathrm{fv} 1, \mathrm{fv} 2, \mathrm{fv} 3\}$. It "rst sends lookup message to feature $f v 1, \mathrm{fv} 2$ and $\mathrm{fv} 3$. fv2 does not exists. A Location Failure message is sent back. $\mathrm{fv} 1$ is managed by object node X. It sends back a list of IDs of all objects having feature $\mathrm{fv} 1$, which is $\{$ guidl $\}$. Similar operation is done for feature $\mathrm{fv} 3$, whose IDs list $\{$ guidl, guid4 $\}$. Client node counts the occurrence of all IDs in all lists and "nds out guidl to be the ID it is looking for. It then sends the payload message to object guid1 using Tapestry location message.

is not found in the network, the client node receives a LocationFailure message, creates a new feature object containing the new object, and publishes it.

For the RouteToApproxObject call, the client node "rst uses Tapestry location to send messages to all feature objects, asking for a list of IDs associated with each feature value. Nodes where these feature objects reside receive these messages, do the lookup in their maps and send back the result. LocationFailure messages are sent back for nonexistent feature objects, and are counted as an empty ID list. The client node counts the occurrence of each GUID in the resulting lists. GUIDs with less than the threshold number of counts are removed. Finally, the message in this call is sent to the remaining object GUIDs An example of executing a RouteToApproxObject call is shown in Figure 4

Note that an analogous system can be implemented on top of a distributed hash table (DHT) abstraction on P2P systems. Instead of routing messages to previously published feature objects, one would retrieve each feature object by doing a get operation, appending the new GUID, and putting the object back using put.

\subsection{Optimizing ADOLR Location}

Our initial description of the RouteToApproxObject operation involves several roundtrips from the client node to nodes where the feature objects are stored. We propose two optimizations here that eliminates a network round-trip, reducing overall latency to that of a normal RouteToObject in a DOLR system at the cost of keeping a small amount of state on overlay nodes. The "rst optimization involves a client node caching the result of translating a feature vector to a GUID. now all future messages to the same feature vector are routing to the cached GUID. 


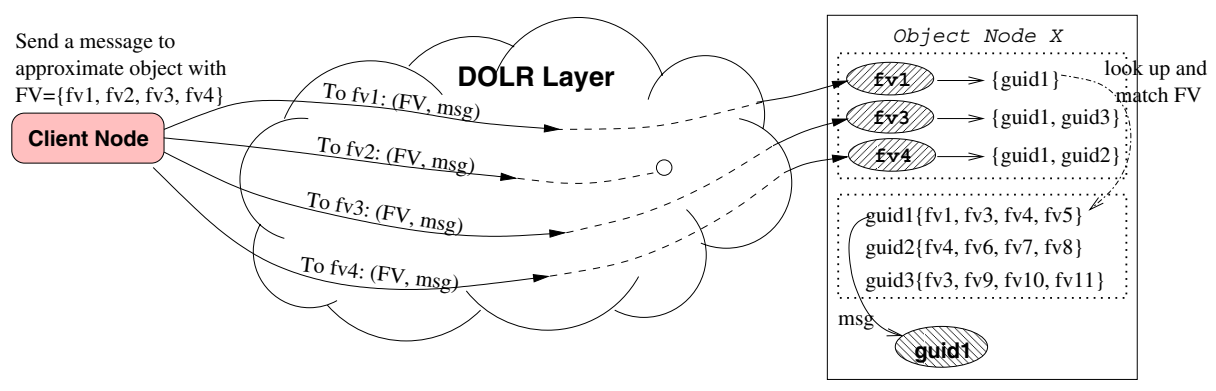

Fig. 5. Optimized ADOLR location. Client node wants to route a message to a feature vector $\{f v 1, f v 2, f v 3, f v 4\}$. It sends message to each identi“er fv1, fv2, fv3, fv4. fv2 doesn॰t exist, so no object node receives this message. When object node $\mathrm{X}$ receives the messages to $\mathrm{fv} 1$, $\mathrm{fv} 3$ and $\mathrm{fv} 4$, it scans its local storage for all IDs matching $\mathrm{fv} 1, \mathrm{fv} 3$ and $\mathrm{fv} 4$, which is guid1. Then, object node $\mathrm{X}$ sends msg to guid1.

The second optimization is more complex, and illustrated in Figure [5] Normally, the client node retrieves a set of feature objects, counts GUID occurrences locally, then routes a message to the resulting GUID(s). The intuition here is that if features are identi“"ed as hashed keys with reasonably low collision rates, each feature will likely only identify a small number (one or two) of objects with that feature. Furthermore, multiple feature objects are likely to be colocated together along with the object they identify, because new feature objects are created by the same node where the object is stored. Another way to look at this is that the feature object is in most cases published at the same time with the object itself by the same node. This implies we can route the application-level message to each feature in the feature vector, and expect it to arrive at the node where the desired object is stored.

The key change here is that any node that is storing a feature object, (a "le providing a mapping from a feature to all GUIDs that share that feature), also stores the feature vectors of each of those GUIDs. Routing a message to a feature vector $\{X, Y, Z\}$ means sending the message to each identi“"er $X, Y$, and $Z$. Each message also includes the entire feature vector were querying for. When a node receives such a message, it immediately scans its local storage for all feature objects matching $X, Y$, or $Z$. For each GUID in these feature objects, the node determines the amount of overlap between its feature vector and the queried feature vector. If the overlap sati“es the query threshold, the message is delivered to that GUID॰s location.

This implies that any of the query messages contains enough information for a node to completely evaluate the ADOLR search on local information. If any locally stored feature objects contain references to matching objects, they can be evaluated immediately to determine if it satis"es the query. Because each message contains all necessary information to deliver the payload to the desired GUID, the set of messages sent to $X, Y$, and $Z$ provide a level of fault-resilience against message loss. Finally, the determination of the desired GUID can occur when the "rst message is received, instead of waiting for all messages to arrive.

The translation from the feature vector to one or more GUIDs occurs in the network, not the client node. This provides signi"cant communication savings. 
Nodes need to keep more state to support this optimization, however. In addition to storing feature objects (that keep the mapping between feature values and GUIDs), they also need to keep track of previously resolved feature vectors in order to drop additional requests for the same feature vector. This state can be stored on a temporary basis, and removed after a reasonable period of time (during which any other requests for the same feature vector should have arrived).

\subsection{Concurrent Publication}

There is one problem with the PublishApproxObject implementation described above. The lookup of feature objects and publication of new feature objects are not atomic. This can result in multiple feature objects for the same feature value being published if more than one node tries to publish an object with this feature value concurrently.

We propose two solutions. First, we can exploit the fact that every object is mapped to a unique root node and serialize the publication on the root node. Every node is required to send a message to the root node of the feature value to obtain a leased lock before publishing the feature object. After the lock is acquired by the "rst node, other nodes trying to obtain it will fail, restart the whole process, and "nd the newly published feature object. This incurs another round-trip communication to the root node.

In a more ef"cient $\bullet$ optimistic $\breve{Z}$ way to solve this problem, the client node always assumes the feature object does not exist in the network. It tries to publish the object without doing a lookup beforehand. When the publication message travels through the network, each node checks whether it knows about an already published feature object with the same feature value. If such an object does exist, some node or at least the root will know about this. The node who detects this then cancels this publication and sends an message to the existing feature object to $\bullet$ merge ̌̌the new information. This process is potentially more ef"cient since con"icts should be rare. In general, the operation is accomplished with a single one-way publication message.

This optimistic approach can easily be implemented on top of DOLRs such as Tapestry using the recently proposed common upcall interface for peer to peer (P2P) overlays [2]. This proposed upcall interface allows P2P applications to override local routing decisions. Speci“cally, a node can •interceptŽ the publication message and handle con"icts as speci“"ed above.

\section{Approximate Text Addressing}

In this section, we present the design for the Approximate Text Addressing facility built on the Approximate DOLR extension, and discuss design decisions for exploring tradeoffs between computational and bandwidth overhead and accuracy.

\subsection{Finding Text Similarity}

Our goal is to ef"ciently match documents distributed throughout the network that share strong similarities in their content. We focus here on highly similar "les, such as modi"ed email messages, edited documents, or news article published on different web sites. 


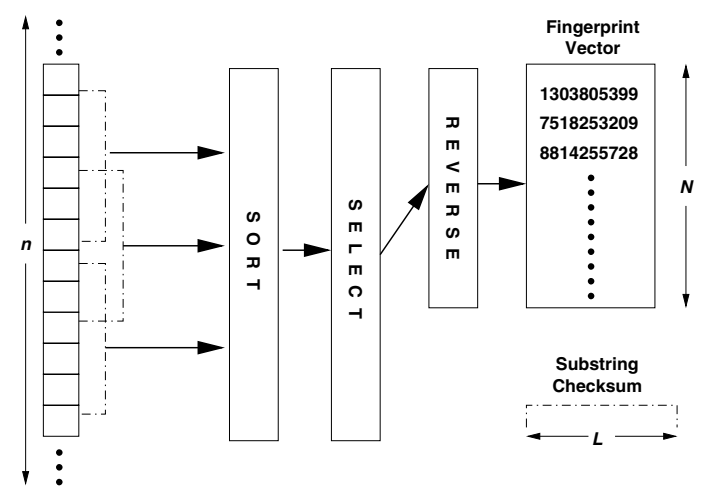

Fig. 6. Fingerprint Vector. A "ngerprint vector is generated from the set of checksums of all substrings of length $L$, post-processed with sort, selection and reverse operations.

The algorithm is as follows. Given a text document, we use a variant of block text "ngerprinting "rst introduced in [7] to generate a set of "ngerprints. The "ngerprint vector of a document is used as its feature vector in publication and location, using the Approximate DOLR layer.

To calculate a block text "ngerprint vector of size $N$ for a text document, we divide the document into all possible consecutive substrings of length $L$. A document of length $n$ characters will have $(n-L+1)$ such strings. Calculating checksums of all such substrings is a fast operation which scales with $n$. We sort the set of all checksums by value, select a size $N$ subset with the highest values, and reverse each checksum by digit (i.e. $123 \Rightarrow 321$ ). This deterministically selects a random set without biasing the ID for pre" $\mathrm{x}$ or numerical routing.

$L$ is a parameterized constant chosen for each application to tune the granularity of similarity matches. For example, a size $L$ of 50 might work well for email, where complete sentences might account for one substring; but less well for source code, where code fragments are often much longer in length. Figure 6 illustrates the "ngerprint process. The calculation is not expensive. Our Java prototype has a processing throughput of $>13 \mathrm{MB} / \mathrm{s}$ for $L=50$ on a $1 \mathrm{Ghz}$ PIII laptop.

\subsection{Trade-offs}

There are obvious trade-offs between network bandwidth used and the accuracy of the search. First, the greater the number of entries $N$ in a vector, the more accurate the match (less false-positives), and also the greater number of parallel lookup requests for each document. Next, the distance each lookup requests travels directly impacts bandwidth consumption on the overall network. ATA-enabled applications 11 can bene"t from exploiting network-locality by matching against similar documents nearby in the network via a DOLR/DHT with object location locality such as Tapestry. Finally, a trade-off exists

\footnotetext{
${ }^{1}$ Some example applications include spam "lters, plagiarism detection and news article clustering.
} 
between the number of publishers (those who indicate they have a particular document), and the resources required for a client to "nd a match in their query. Bandwidth and accuracy can be tuned by placing a Time-to-Live (TTL) "eld on the lookup query, constraining the scope of query messages. Clients who fail to "nd a match may publish their own documents, improving lookup performance for other clients. These are explored in detail in Section 6 .

\section{Decentralized Spam Filtering}

Spam, or unsolicited email, wastes time and valuable network resources, causing headaches for network administrators and home users alike. Currently the most widelydeployed spam "Itering systems scale to a university- or company- wide network, and use keyword matching or source address matching [13]. Although easy to deploy and manage, these systems often walk a "ne line between letting spam through and blocking legitimate emails. Our observation is that human recognition is the only fool-proof spam identi"cation tool. Therefore, we propose a decentralized spam "Iter that pools the collective spam recognition results of all readers across a network.

There already exist centralized collaborative spam "Itering systems, such as SpamNet [14], which claims to be peer-to-peer but actually uses a Napster-like architecture. To our knowledge ours is the "rst attempt to build a truly decentralized collaborative spam "Itering system. Compared to alternative university-wide centralized collaborated designs, the most important bene" $t$ of our wide-area decentralized design lies in the fact that the effectiveness of the system grows with the number of its users. In such a system with huge number of users world-wide, it is highly probable that every spam email you receive has been received and identi"ed by somebody else before because of the large number of users. The deterministic behavior of DOLR systems will prove useful, because when any single peer publishes information about a speci"c email, that piece of information can be deterministically found by all clients. Therefore we can expect this system to be more responsive to new spam than systems in which different nodes publish/exchange spam information at certain intervals, such as [3]. Additionally, decentralized systems provide higher availability and resilience to failures and attacks than similar centralized solutions such as SpamNet.

\subsection{Basic Operation}

The decentralized spam "Itering system consists of two kinds of nodes, user agents and peers. User agents are extended email client programs that users use. They query peers when new emails are received and also send useres feedback regarding whether a certain email is or is not spam to peers. A peer is a piece of long-running software that is installed typically on a university, department or company server that speaks to other peers worldwide and forms a global P2P network.

When an email client receives a message from the server, the user agent extracts the body of the mail, drops format artifacts like extra spaces and HTML tags, generates a "ngerprint vector, and sends it to a peer in the DOLR system. The peer in turn queries the network using the Approximate DOLR API to see if information on the email has 
been published. If a match is found, and it indicates the email is spam, the email will be "led separately or discarded depending on user preference. Otherwise, the message is delivered normally. If the user marks a new message as spam, the user agent marks the document, and tells the peer to publish this information into the network.

\subsection{Enhancements and Optimizations}

The basic design above allows human identi“cation of spam to quickly propagate across the network, which allows all users of the system to bene"t from the feedback of a few. There are several design choices and optimizations which will augment functionality and reduce resource consumption.

Our "ngerprint vectors make reverse engineering and blocking of unknown emails very dif"cult. With the basic system, however, attackers can block well known messages (such as those from group mailing lists). We propose to add a voting scheme on top of the publish/search model. A count of positive and negative votes is kept by the system, and each user can set a threshold value for discarding or "ling spam using the count as a con"dence measure. A central authority controls the assignment and authentication of user identities. A user agent is required to authenticate itself before being able to vote for or against an email. Thus we can restrict the number of votes a certain user agent can perform on a certain email.

Another type of attack is for spammers to "nd arbitrary text segments with checksum values more likely to be selected by the "ngerprint selection algorithm. By appending such •preferredŽ segments to their spam emails, spammers can " $x$ the resulting email "ngerprint vectors to attempt to avoid detection. Note that this attack can only succeed if a continuous stream of unique text segments are generated and an unique segment is appended to each spam message. This places a signi"cant computational overhead on the spammer that scales with the number of spam messages sent. Additionally, mail clients can choose randomly from a small set of "ngerprint calculation algorithms. Different "ngerprinting methods can include transforming the text before calculating the checksums, changing the checksum method, or changing the "ngerprint selection method. To circumvent this, the spammer would need to "rst determine the set of "ngerprint algorithms, and then append a set of preferred segments, each segment overcoming a known selection algorithm. While different "ngerprint algorithms generate distinct spam signatures for the same spam, partitioning the user population and reducing the likelihood of a match, it also requires signi"cantly more computational overhead to overcome.

Optimizations can be made for centralized mail servers to compute "ngerprint vectors for all incoming messages. These vectors can be compared locally to identify •popularŽmessages, and lookups performed to determine if they are spam. Additionally, the server can attach precomputed "ngerprint vectors and/or spam "Itering results as custom headers to messages, reducing local computation, especially for thin mail clients such as PDAs.

\section{Evaluation}

In this section, we use a combination of analysis, experimentation on random documents and real emails to validate the effectiveness of our design. We look at two aspects 
of "ngerprinting, robustness to changes in content and false positive rates. We also evaluate "ngerprint routing constrained with time-to-live (TTL) "elds, tuning the tradeoff between accuracy and network bandwidth consumption.

\subsection{Fingerprint on Random Text}

We begin our evaluation by examining the properties of text "ngerprinting on randomly generated text. In particular, we examine the effectiveness of "ngerprinting at matching text after small modi"cations to their originals, and the likelihood of matching unrelated documents (false positive rate).

Robustness to Changes in Content. We begin by examining the robustness of the " $n$ gerprint vector scheme against small changes in a document, by measuring the probability a "ngerprint vector stays constant when we modify small portions of the document. We " $\mathrm{x}$ the "ngerprint vector size, and want to measure the robustness against small changes under different threshold constants (THRES).

In experiments, we take 2 sets of random text documents of size $1 \mathrm{~KB}$ and $5 \mathrm{~KB}$, which match small- and large-sized spam messages respectively, and calculate their "ngerprint vectors before and after modifying 10 consecutive bytes. This is similar to text replacement or mail merge schemes often used to generate differentiated spam. We measure the probability of at least THRES out of $|F V|$ "ngerprints matching after modi"cation as a function of threshold (THRES) and the size of the document $(1 \mathrm{~KB}$ or $5 \mathrm{~KB})$. Here, "ngerprint vector size is $10,|F V|=10$. We repeat that experiment with a modi"cation of 50 consecutive bytes, simulating the replacement of phrases or sentences and "nally modifying 5 randomly placed words each 5 characters long.

In addition to the simulated experiments, we also developed a simple analytical model for these changes based on basic combinatorics. We present this model in detail in Appendix A. For each experiment, we plot analytical results predicted by our model in addition to the experimental results.

In Figure 7, we show for each scenario experimental results gathered on randomized text "les, by comparing "ngerprint vectors before and after modi“cations. From Figure7, we can see the model in Appendix A predicts our simulation data almost exactly under all three patterns of modi“"cation. More speci"cally, modifying 10 characters in the text only impacts 1 or 2 "ngerprints out of 10 with a small probability. This means setting any matching threshold below 8 will guarantee near $100 \%$ matching rate. When we increase the length of the change to 50 characters, the results do not change signi"cantly, and still guarantee near perfect matching with thresholds below 7 . Finally, we note that multiple small changes (in the third experiment) have the most impact on changing "ngerprint vectors. Even in this case, setting a threshold value around 5 or less provides a near perfect matching rate.

Avoiding False Positives. In addition to being robust under modi“cations, we also want "ngerprint vectors to provide a low rate of false positives (where unrelated documents generate matching entries in their vectors). In this section, we evaluate "ngerprint vectors against this metric with simulation on random text documents. In Section 6.2, we present similar tests on real email messages. 

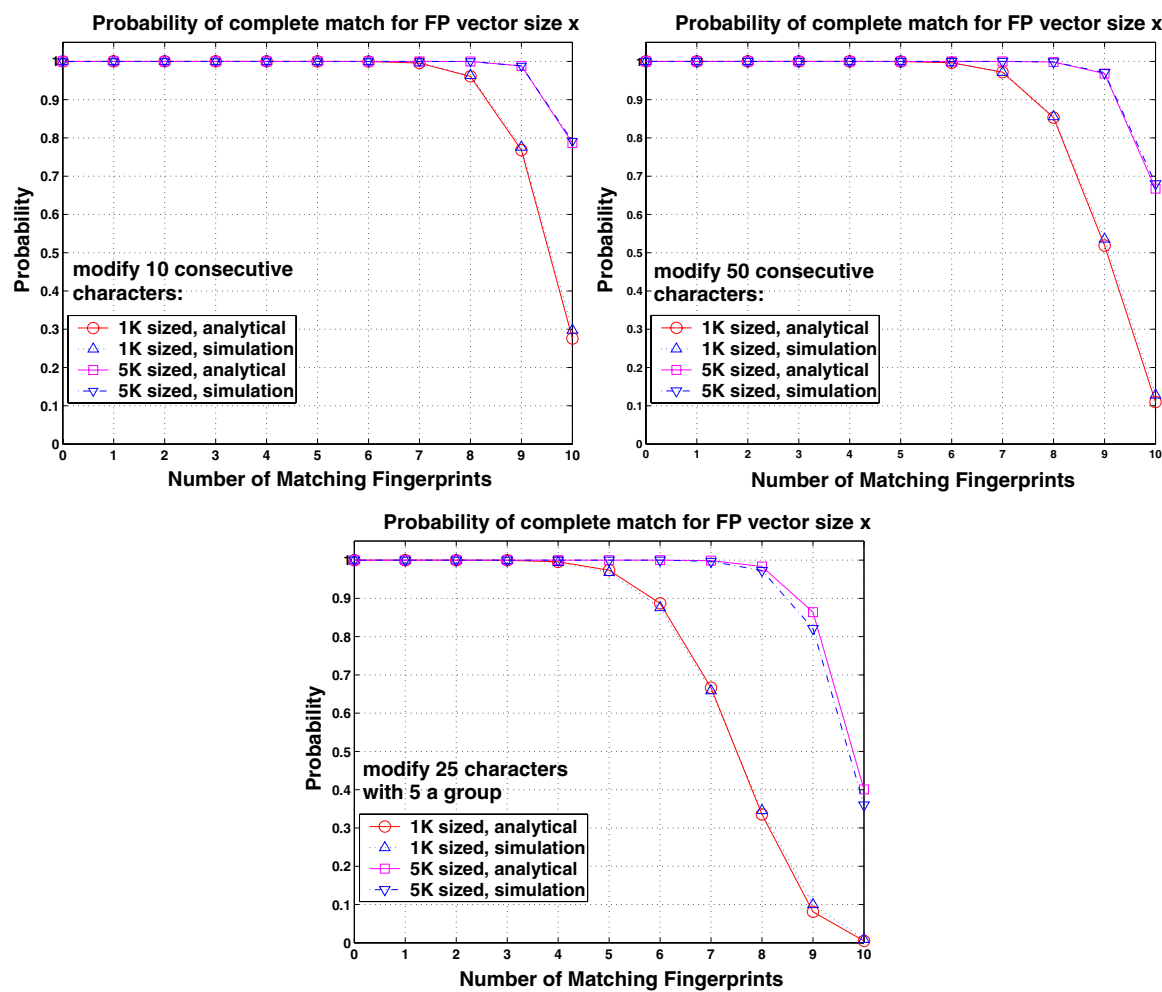

Fig. 7. Robustness Test (Experimental and Analytical). The probability of correctly recognizing a document after modi“cation, as a function of threshold. $|F V|=10$.

First, we generate 100,000 random text "les and "nd document pairs that match 1 out of 10 "ngerprint entries. This experiment is done for different "le sizes ranging from $1 \mathrm{~KB}$ to $64 \mathrm{~KB}$. Figure 8 shows the resulting false positive rate versus the "le size. While the results for one "ngerprint match are already low, they can be made statistically insigni"cant by increasing the "ngerprint matches threshold (THRESH) for a $\bullet$ document match.Ž Out of all our tests $\left(5 \times 10^{9}\right.$ pairs for each "le size $)$, less than 25 pairs of "les ("le size $>32 \mathrm{~K}$ ) matched 2 "ngerprints, no pairs of "les matched more than 2 "ngerprints. This result, combined with the robustness result, tells us that on randomized documents, a threshold from 2 to 5 "ngerprints gives us a matching mechanism that is both near-perfect in terms of robustness against small changes and absence of false positives.

\subsection{Fingerprint on Real Email}

We also repeat the experiments in Section 6.1 on real emails. We collected 29996 total spam email messages from http : / / www . spamarchive.org. Histogram and CDF representations of their size distribution are shown in Figure 9 


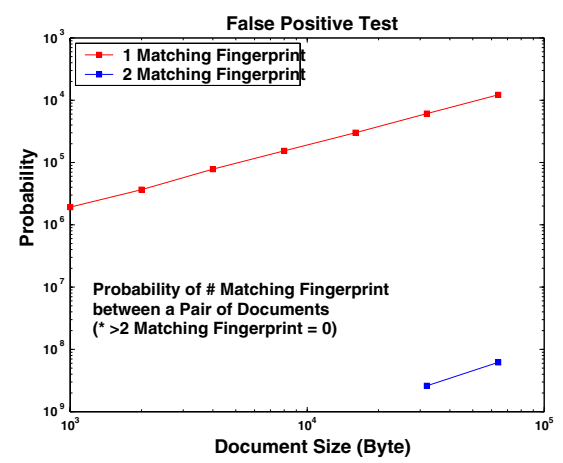

Fig. 8. False Positives. The probability of two random text "les matching $i(i=1,2)$ out of 10 "ngerprint vectors, as a function of "le size.

Table 1. Robustness Test on Real Spam Emails. Tested on 3440 modi"ed copies of 39 emails, 5629 copies each. $|F V|=10$.

\begin{tabular}{ccccc}
\hline \multicolumn{6}{c}{ THRES Detected } & Failed & Total & Succ. \% \\
\hline 3 & 3356 & 84 & 3440 & 97.56 \\
4 & 3172 & 268 & 3440 & 92.21 \\
5 & 2967 & 473 & 3440 & 86.25 \\
\hline
\end{tabular}

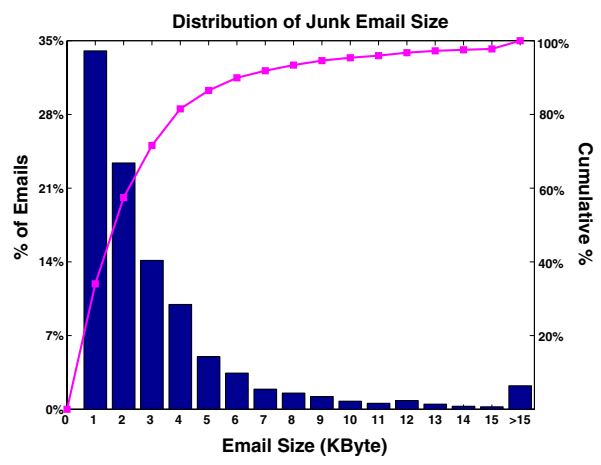

Fig. 9. Spam Mail Sizes. Size distribution of the 29996 spam email messages used in our experiments, using both histogram and CDF representations.

Table 2. False Positive Test on Real Spam Emails. Tested on 9589(normal) × 14925 (spam) pairs. $|F V|=10$.

\begin{tabular}{|c|c|c|}
\hline \multicolumn{3}{|c|}{ Match FP \# of Pairs Probability } \\
\hline 1 & 270 & $1.89 \mathrm{e}-6$ \\
\hline 2 & 4 & $2.79 \mathrm{e}-8$ \\
\hline$>2$ & 0 & 0 \\
\hline
\end{tabular}

In order to get an idea of whether small modi"cations on spam email is a common practice of spammers, we used a variant of our "ngerprint techniques to fully categorize the email set for uniqueness. We personally con"rmed the results. We found that, out of all these 29996 junk emails, there are:

- 14925 unique junk emails.

- 9076 modi“"ed copies of 4585 unique ones.

- 5630 exact copies of the unique ones.

From statistics above, we can see that about 1/3 junk emails have modi“ed version(s), despite that we believe the collectors of the archive have already strive to eliminate duplicates. This means changing each email they sent is really a common technique used by spammers, either to prevent detection or to misdirect the end user.

We did the robustness test on 3440 modi“"ed copies of 39 most •popularŽ junk emails in the archive, which have $5-629$ copies each. The standard result is human processed and made accurate. The "ngerprint vector size is set to $10,|F V|=10$. We vary threshold of matching "ngerprint from 3 to 5 , and collect the detected and failed number. Table 1 shows the successful detection rate with $T H R E S=3,4,5$ are satisfying.

For the false positive test, we collect 9589 normal emails, which is compose of about half from newsgroup posts and half from personal emails of project members. Before doing the experiment, we expect collisions to be more common, due to the use of common words and phrases in objects such as emails. We do a full pair-wise "ngerprint 
match (vector size 10) between these 14925 unique spam emails and 9589 legitimate email messages. Table 2 shows that only 270 non-spam email messages matched some spam message with 1 out of 10 "ngerprints. If we raise the match threshold $T$ to 2 out of 10 "ngerprints, only 4 matches are found. For match threshold more than 2, no matches are found. We conclude that false positives for threshold value $T>1$ are very rare $\left(\sim 10^{-8}\right)$ even for real text samples.

\subsection{Efficient Fingerprint Routing w/ TTLs}

We want to explore our "ngerprint routing algorithms in a more realistic context. Specifically, we now consider the additional factor mark rate, which is the portion of all users in the network that actively report a particular spam. A user who •marksŽ a spam message actives publishes this fact, thereby registering that opinion with the network. For example, a $10 \%$ mark rate means that $10 \%$ of the user population actively marked the same message as spam.

To simulate the trade-off between bandwidth usage, •mark ̌̌ rate, and search success rate, we simulate the searching of randomly generated "ngerprints on transit-stub networks, and vary the required number of overlay hops to "nd a match, as well as the mark rate. We assume users marking the spam are randomly distributed. With an ef"cient DOLR layer, the more users who mark a document as spam, the fewer number of hops we expect a query to travel before "nding a match. We can set a TTL value on queries to conserve bandwidth while maintaining a reasonably high search success rate.

We performed experiments on 8 transit stub topologies of 5000 nodes, latency calibrated such that the network diameter is 400ms. Each Tapestry network has 4096 nodes, and each experiment was repeated with 3 randomized overlay node placements. By aggregating the data from all placements and all topologies, we reduced the standard deviation below 0.02 ( 0.01 for most data points).

The results in Figure 10 show the expected latency and success probability for queries as a function of the number of hops allowed per query (TTL). Since there is a high correlation between the TTL value and the network distance traveled in ms, we plot both the TTL used and the associated network distance. For example, we see that queries with TTL of 2 on these topologies travel a distance of approx. 60ms. Further, at $10 \%$ publication rate, we expect those queries to be successful $75 \%$ of the time. We note that a Time-to-Live value of 3 overlay hops results in a high probability of "nding an existing document even if it has only been reported by a small portion of the participating nodes $(2-5 \%)$.

\section{Related Work}

There has been a large amount of recent work on structured peer to peer overlays [18 5, 11,15 10,84]. Recent work [2] has tried to clarify the interfaces these protocols export to applications, including distributed hash tables (DHTs) and decentralized object location and routing (DOLRs) layers. While our proposal is designed for DOLR systems, it can also be implemented on top of DHTs with minor modi“"cations. Furthermore, protocols like Tapestry that use network proximity metrics to constrain network traf"c will bene"t the most from our performance optimizations. 


\section{Success Probability vs. Expected Latency and TTL}

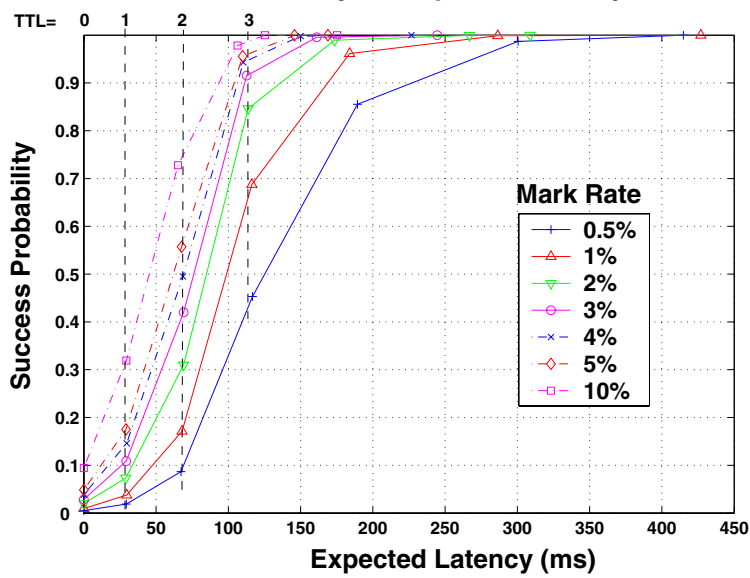

Fig. 10. Finding an Ideal TTL. A graph that shows, for a •markedŽ document, the correlation between TTL values on queries, probability of a successful search, and percentage of nodes in the network who •markedŽit.

Recent work [6] discusses the feasibility of doing keyword-based web search in structured P2P networks, which can be thought of as an instantiation of our ADOLR proposal applied to text documents with keywords used as features. Both their scheme and our work use inverted indices of keywords/features assigned to different nodes and maintained using structured overlay location and routing primitives. Finally, this work tries to gauge feasibility, rather than to propose any speci“c implementation.

In the context of approximate text addressing, centralized text similarity search is a well-studied problem. Comprehensive discussion can be found in [17]. It includes discussion about using "n-grams" to do similarity search using exact search facility. One speci“"c technique within this category [7] forms the basis of our approach of using checksum based "ngerprints.

In [1], Broder examined the probability of two different strings colliding to an identical single "ngerprint. In contrast, we focus on the collision probability of entire "ngerprint vectors. In Appendix A, we also consider the probability of changes in a "ngerprint vector under different document modi"cation patterns.

Many spam "ltering schemes have been proposed and some deployed. Schemes based on hashing and fuzzy hashes [1614,3], including our proposal, are collaborative and utilize community consensus to "Iter messages. These systems include two main components: one or more hash functions to generate digests of email messages, and a repository of all known digests and whether the corresponding emails are spam. Our system differs from others in this group in that the digest repository is fully decentralized, and queries are deterministic by default (i.e. all existing results will be found no matter where it is). This ensures both scalability and accuracy.

Another big family of spam "ltering schemes are machine learning-based [129]. These schemes "lter incoming messages based on symptoms or trails of spam emails identi“ed explicitly or implicitly by the training process. They can be personalized 
according to user preferences and email content and therefore perform well on client machines. However, because the "Iters these systems use are only based on per-user local information and do not allow cross-user collaboration, they have dif"culty in identifying new spam emails that are very different from those seen before by the local user.

\section{Ongoing and Future Work}

We have implemented the basic Approximate DOLR and Approximate Text Addressing prototype on a Java implementation of Tapestry, and are exploring additional optimizations and extensions. A prototype of the proposed P2P spam "Itering system, SpamWatch, is implemented and available, including a per-node component implemented as a Tapestry application and the user interface implemented as a Microsoft Outlook plug-in 2 . One direction for future work is to deploy SpamWatch as a longrunning service, both to provide a valuable service and also to collect valuable trace data. We are also considering extending the system to handle predicate queries.

In conclusion, we proposed the design of an approximate location extension to DOLR systems and described an Approximate Text Addressing facility for text-based objects. We discuss issues of data consistency and performance optimizations in the system design, and present a decentralized spam "Itering system as a key application. We validate our designs via simulation and real data, and show how to tune the "ngerprint vector size and query TTL to improve accuracy, reduce bandwidth usage and query latency, all while keeping a low false positive rate.

\section{References}

1. Broder, A. Z. Some applications of rabines "ngerprint method. In Sequences II: Methods in Communications, Security, and Computer Science, R. Capocelli, A. D. Santis, and U. Vaccaro, Eds. Springer Verlag, 1993, pp. 143...152.

2. Dabek, F., Zhao, B. Y., Druschel, P., Kubiatowicz, J., and Stoica, I. Towards a common API for structured P2P overlays. In Proceedings of IPTPS (Berkeley, CA, February 2003).

3. Distributed checksum clearinghouse. http://www.rhyolite.com/anti-spam/dcc/

4. Harvey, N. J. A., Jones, M. B., Saroiu, S., Theimer, M., and Wolman, A. Skipnet: A scalable overlay network with practical locality properties. In Proceedings of USITS (Seattle, WA, March 2003), USENIX.

5. Hildrum, K., Kubiatowicz, J. D., Rao, S., and ZhaO, B. Y. Distributed object location in a dynamic network. In Proceedings of ACM SPAA (Winnipeg, Canada, August 2002).

6. Li, J., Loo, B. T., Hellerstein, J., KaAshoek, F., Karger, D. R., and Morris, R. On the feasibility of peer-to-peer web indexing and search. In 2nd International Workshop on Peer-to-Peer Systems (Berkeley, California, 2003).

7. Manber, U. Finding similar "les in a large "le system. In Proceedings of Winter USENIX Conference (1994).

8. Maymounkov, P., And Mazieres, D. Kademlia: A peer-to-peer information system based on the XOR metric. In Proceedings of 1st International Workshop on Peer-to-Peer Systems (IPTPS) (Cambridge, MA, March 2002).

\footnotetext{
${ }^{2}$ Fully functional prototypes of the ATA layer and spam "Iter are available for download at http://www.cs.berkeley.edu/ zf/spamwatch
} 
9. Mozilla spam "ltering. http://www.mozilla.org/mailnews/spam.html.

10. Ratnasamy, S., Francis, P., Handley, M., KarP, R., and Schenker, S. A scalable contentaddressable network. In Proceedings of SIGCOMM (August 2001).

11. Rowstron, A., AND Druschel, P. Pastry: Scalable, distributed object location and routing for large-scale peer-to-peer systems. In Proceedings of IFIP/ACM Middleware 2001 (November 2001).

12. Sahami, M., Dumais, S., Heckerman, D., and Horvitz, E. A bayesian approach to "ltering junk email. In AAAI Workshop on Learning for Text Categorization (Madison, Wisconsin, July 1998).

13. Spamassassin. http://spamassassin.org.

14. Spamnet. http://www. cloudmark.com

15. Stoica, I., Morris, R., Karger, D., KaAshoek, M. F., and Balakrishnan, H. Chord: A scalable peer-to-peer lookup service for internet applications. In Proceedings of SIGCOMM (August 2001).

16. Vipuløs razor. http://razor. sourceforge.net/.

17. Witten, I. H., Moffat, A., AND Bell, T. C. Managing Gigabytes: Compressing and Indexing Documents and Images, second ed. Morgan Kaufmann Publishing, 1999.

18. Zhao, B. Y., Kubiatowicz, J. D., ANd JosePh, A. D. Tapestry: An infrastructure for faulttolerant wide-area location and routing. Tech. Rep. UCB/CSD-01-1141, U.C. Berkeley, April 2001.

\section{A Analysis of Robustness of Text Fingerprinting}

Here we give mathematical analysis of how to compute the probability distribution of number of unchanged "ngerprints of a text document after small modi"cations.

We de"ne:

$D$ : the original document

$D^{\prime}$ : the original document after modi“cations

$L$ : the document is divided in consecutive substrings of length $L$ characters

$A$ : the set of checksums calculated from all substrings in $D$

$B$ : the set of checksums calculated from all substrings in $D^{\prime}$

$X: A-B$, checksums from $D$ which are not present in checksums of $D^{\prime}$

$Y: B-A$, checksums from $D^{\prime}$ not present in original checksums of $D$

$F P(A)$ : the "ngerprint vector generated from checksums of $D$, such that $F P(A) \subseteq A$, $|F P(A)|=N$

$F P(B)$ : the "ngerprint vector generated from checksums of $D^{\prime}$, such that $F P(B) \subseteq B$, $|F P(B)|=N$

$|S|$ : if $S$ is a set or vector, $|S|$ represents the size of $S$

$z:|F P(B)-F P(A)|$, number of checksums in new "ngerprint vector which are not in the old "ngerprint vector

Refer to Figure 11 for an illustration of $X, Y, A$ and $B$.

Let's de"ne $P_{r}(x)$ as the probability that $x$ out of $N$ checksums in $F P(A)$ are obsolete, that is, not in $B$; de"ne $P_{r}(y)$ as the probability that $y$ out of $N$ checksums in $F P(B)$ are newly generated, that is, not in $A$. We have:

$$
P_{r}(x)=P_{r}(|F P(A) \cap X|=x)=\frac{\left(\begin{array}{c}
|X| \\
x
\end{array}\right) \times\left(\begin{array}{c}
|A|-|X| \\
N-x
\end{array}\right)}{\left(\begin{array}{c}
|A| \\
N
\end{array}\right)}
$$




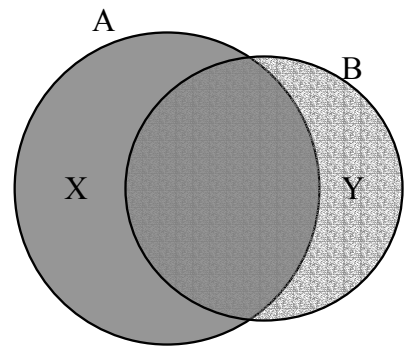

Fig. 11. Relationship between $X, Y, A$ and $B$.

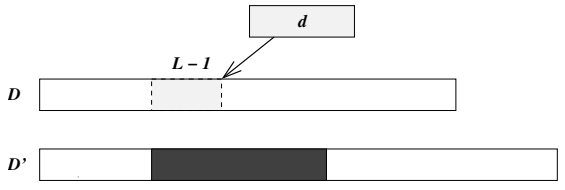

Fig. 13. Insert $d$ chars.

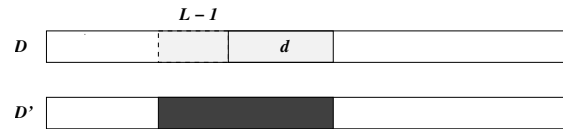

Fig. 12. Update d chars.

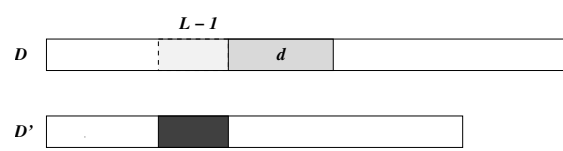

Fig. 14. Delete d chars.

$$
P_{r}(y)=P_{r}(|F P(B) \cap Y|=y)=\frac{\left(\begin{array}{c}
|Y| \\
y
\end{array}\right) \times\left(\begin{array}{c}
|B|-|Y| \\
N-y
\end{array}\right)}{\left(\begin{array}{c}
|B| \\
N
\end{array}\right)}
$$

If:

1. $(N-x)+y<N$ (that is, $x>y)$ : $F P(B)$ is composed of $(N-x)$ checksums from $F P(A)$, y checksums from newly generated set $Y$, and others from $A \cap B$. That is, the $y$ checksums from $Y$ and others from $A \cap B$ are the new checksums in $F P(B)$ since $F P(A)$. Then, $z=N-(N-x)=x$.

2. $(N-x)+y \geq N$ (that is, $y \geq x): F P(B)$ is composed of $y$ checksums from $Y$, other checksums from $F P(A)-X$. That is, the $y$ checksums from $Y$ are new checksums in $F P(B)$ since $F P(A)$. Then, $z=y$.

So, when $x>y, z=x$; when $y \geq x, z=y$. That is, $z=\max (x, y)$. Then,

$$
P_{r}(z)=P_{r}(y=z) \sum_{i=0}^{z} P_{r}(x=i)+P_{r}(x=z) \sum_{i=0}^{z} P_{r}(y=i)-P_{r}(x=z) P_{r}(y=z)
$$

Let•s de"ne $P(|F P(A) \cap F P(B)| \geq k)$ to be the probability that at least $k$ checksums are in common between "ngerprint vector of new document and of old document. We have:

$$
P_{r}(|F P(A) \cap F P(B)| \geq k)=P_{r}(|F P(B)-F P(A)| \leq N-k)=\sum_{i=0}^{N-k} P_{r}(z=i)
$$

Knowing of $|X|$ and $|Y|$, we can apply results in equation (1)-(3) to equation (4), and then get the probability of the number of unchanged "ngerprints after modi"cation of the document. 
While $|X|$ and $|Y|$ are related to modi"cation pattern, we can further consider how to get $|X|$ and $|Y| . X=\bigcup X_{i}$ and $Y=\bigcup Y_{i}$, where $X_{i}$ and $Y_{i}$ are changes made to checksums because of one modi"cation operation $i$.

We have three types of operations:

Update d characters : $\left|X_{i}\right|=L-1+d,\left|Y_{i}\right|=L-1+d$. This is illustrated in Figure 12.

Insert d characters : $\left|X_{i}\right|=L-1,\left|Y_{i}\right|=L-1+d$. This is illustrated in Figure 13, Delete d characters : $\left|X_{i}\right|=L-1+d,\left|Y_{i}\right|=L-1$. This is illustrated in Figure 14. $X$ equals the union of each $X_{i}$ and $Y$ equals the union of each $Y_{i}$. So, if there is only one modi"cation, we can exactly compute $|X|$ and $|Y|$. If there are more than one modi"cation, $|X|$ ranges from $\max _{i}\left|X_{i}\right|$ to $\sum_{i}\left|X_{i}\right|,|Y|$ ranges from $\max _{i}\left|Y_{i}\right|$ to $\sum_{i}\left|Y_{i}\right|$. We can compute approximate average $|X|$ and $|Y|$ for a speci"c pattern of modi“"cation operations according to equations above.

Thus, we can use equation (4) to compute the probability distribution of number of unchanged "ngerprints in "ngerprint vector. 